\title{
Investigations on the flutter properties of supersonic panels with different boundary conditions
}

\author{
Zhi-Guang Song • Feng-Ming Li
}

Received: 25 July 2013 / Revised: 25 October 2013 / Accepted: 4 November 2013 / Published online: 22 November 2013

(C) Springer-Verlag Berlin Heidelberg 2013

\begin{abstract}
Aeroelastic characteristics of supersonic panels with different boundary conditions are analyzed. The classical thin plate theory is used in the structural modeling. The unsteady aerodynamic pressure is evaluated using the supersonic piston theory. To formulate the governing equation of motion for the aeroelastic structural system, Hamilton's principle is carried out. The assumed mode method (AMM) and the finite element method are used to obtain the discrete equation of motion. Frequency-domain method is conducted to analyze the aeroelastic performances of the panel with different boundary conditions. The special flutter properties of panels with elastically restrained boundary condition are also investigated. The accuracy of the AMM in the flutter analysis of panels with different constraints is researched. Aeroelastic characteristics of the panels with different boundary conditions are also investigated.
\end{abstract}

Keywords Supersonic panel - Flutter characteristic . Piston theory - Assumed mode method · Finite element method $\cdot$ Elastically restrained boundary

\section{Introduction}

Flutter, a kind of self-excited oscillation, is caused by the coupling effect of the aerodynamic load, the elastic force as well as the inertial force of the structure. Panel flutter as one

Z.-G. Song · F.-M. Li

School of Astronautics, Harbin Institute of Technology,

P.O. Box 137, Harbin 150001, China

F.-M. Li (ه)

College of Mechanical Engineering, Beijing University of Technology,

Beijing 100124, China

e-mail: fmli@bjut.edu.cn kind of the flutter can be generated when a panel is exposed to airflow along its surface. Many literatures have studied the characteristics of the panel flutter.

Dowell [1-3] investigated the nonlinear aeroelastic stability of two- and three-dimensional plates using the Galerkin's method. The mode convergence was analyzed in these works. It is noted that, to get a convergent solution for LCO of an aeroelastic rectangular panel, at least six orders of mode must be used. For complex structures, more modes were needed. Guo and Mei [4] introduced the aeroelastic modes in the analysis of nonlinear panel flutter considering thermal effect. The results showed that the use of aeroelastic modes could reduce the number of coupled nonlinear modal equations significantly. Kouchakzadeh et al. [5] conducted the panel flutter analysis of general laminated composite panels. In the investigation, Galerkin's method was used to reduce the governing equations to a set of nonlinear ordinary differential equations. Also based on the Galerkin's method, Navazi and Haddadpour [6] investigated the aeroelastic properties of two- and three-dimensional functionally graded panels. Li and Song [7-10] studied the aeroelastic behaviors and active flutter control of beams and laminated panels using the AMM.

In the panel flutter analysis, there are also several literatures having applied the FEM. Gray et al. [11] investigated the flutter properties of nonlinear two-dimensional panel with large amplitude using the FEM. Zhou et al. [12] studied the flutter characteristics of nonlinear composite panels considering the thermal effect using the FEM. Also based on the FEM, Han and Yang [13] carried out the investigation on the supersonic nonlinear panel flutter. In the analysis, high-order triangular plate finite element is applied. Li et al. [14] studied the flutter and thermal postbuckling suppression of a large amplitude nonlinear panel using the FEM. Koo and Hwang [15] performed a study of flutter characteristics for composite panels with structural damping. The FEM was used in the 
modeling of equation of motion. Moon et al. [16,17] studied the active optimal flutter control of composite panels using the FEM. Xue and Mei [18] carried out a study on the flutter behaviors of two-dimensional nonlinear panels with thermal effect using the FEM. Li and Song [19] investigated the aerothermoelastic characteristics of laminated panels using the FEM.

It is noted from the above review, most of the investigations on the panel flutter focus on the fully simply supported panels, and few literatures have studied the aeroelastic characteristics of supersonic panels with other different boundary conditions. Moreover, in our recent study [19], it is found that the AMM will produce errors in the aeroelastic analysis of SCSC (simply supported on two opposite edges and clamped on the other two opposite edges) panels. Inspired from the above analysis, in this paper, the aeroelastic performances of panels with different boundary conditions are further studied. The classical thin plate theory is used in the structural modeling. The unsteady aerodynamic pressure is evaluated using the supersonic piston theory. Hamilton's principle is applied to formulate the equation of motion, and the AMM and FEM are used to obtain the discrete equation of motion. Frequency domain method is used to analyze the flutter behavior of the structure. The special flutter properties of panels with elastically restrained boundary condition are also researched. The accuracy of the AMM in the aeroelastic analysis for panels with different constraints is analyzed.

\section{Formulation for the equation of motion}

The supersonic isotropic panel is shown in Fig. 1. The length, width and thickness of the panel are $a, b$ and $h$. The panel investigated here is thin, so the classical thin plate theory is applied. The displacement fields of the structure are given as

$u=-z \frac{\partial w}{\partial x}, \quad v=-z \frac{\partial w}{\partial y}, \quad w=w$

where $u$ and $v$ are the in-plane displacements along the $x$ and $y$ directions, and $w$ is the transverse displacement along the

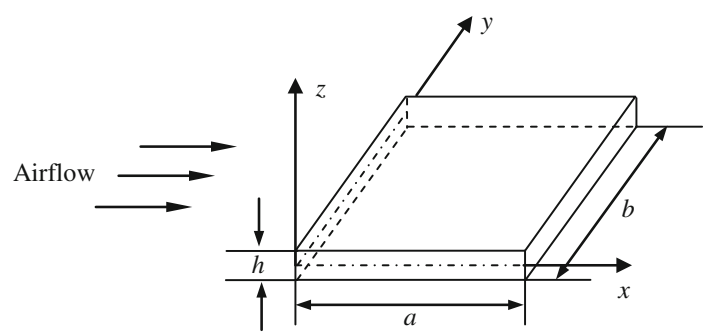

Fig. 1 Schematic diagram of the panel in the supersonic flow $z$ direction. The strain-displacement relation is given as

$\boldsymbol{\varepsilon}=z\left[\begin{array}{lll}-\frac{\partial^{2} w}{\partial x^{2}} & -\frac{\partial^{2} w}{\partial y^{2}} & -2 \frac{\partial^{2} w}{\partial x \partial y}\end{array}\right]^{\mathrm{T}}=z \boldsymbol{\kappa}$,

where $\boldsymbol{\varepsilon}=\left[\varepsilon_{x}, \varepsilon_{y}, \varepsilon_{x y}\right]^{\mathrm{T}}$ is the strain vector, and $\boldsymbol{\kappa}$ is the bending curvature vector. The constitutive equation of the isotropic panel is given as

$\boldsymbol{\sigma}=\left[\begin{array}{l}\sigma_{x} \\ \sigma_{y} \\ \tau_{x y}\end{array}\right]=\left[\begin{array}{ccc}Q_{11} & Q_{12} & 0 \\ Q_{21} & Q_{22} & 0 \\ 0 & 0 & Q_{66}\end{array}\right]\left[\begin{array}{l}\varepsilon_{x} \\ \varepsilon_{y} \\ \gamma_{x y}\end{array}\right]=\boldsymbol{Q} \boldsymbol{\varepsilon}$,

where $\sigma$ is the stress vector, $Q$ is the stiffness matrix, in which $Q_{11}=Q_{22}=E /\left(1-v^{2}\right), Q_{12}=Q_{21}=\mathrm{E} v /\left(1-v^{2}\right)$ and $Q_{66}=E /[2(1+v)]$, where $E$ and $v$ are the Young's modulus and Poisson's ratio.

The equation of motion is formulated using the Hamilton's principle which is written as

$\int_{t_{1}}^{t_{2}}[\delta(T-U)+\delta W] \mathrm{d} t=0$,

where $T, U$ and $\delta W$ are the kinetic energy, strain energy and virtual work done by the external load.

In order to obtain the discrete equation of motion of the supersonic panel, finite element method (FEM) and the assumed mode method (AMM) are used in this study, and the simulation results of these two methods are compared.

In the FEM, the four-node rectangular Kirchhoff plate element is selected. There are three degrees of freedom $\left(w, w_{, x}, w, y\right)$ in each of the four nodes. The element nodal displacement vector is expressed as

$$
\begin{array}{r}
\boldsymbol{w}_{e}=\left[w_{1}, w_{1, x}, w_{1, y}, w_{2}, w_{2, x}, w_{2, y},\right. \\
\left.w_{3}, w_{3, x}, w_{3, y}, w_{4}, w_{4, x}, w_{4, y}\right]^{\mathrm{T}} .
\end{array}
$$

The element displacement $w$ can be interpolated by the interpolation functions as

$w=\left[\begin{array}{lllll}\zeta_{1} & \zeta_{2} & \zeta_{3} & \ldots & \zeta_{12}\end{array}\right]^{\mathrm{T}} \boldsymbol{w}_{b e}=\zeta^{\mathrm{T}} \boldsymbol{w}_{b e}$,

where $\zeta$ is the interpolation function vector. The kinetic and strain energies of the element can be calculated by

$$
\begin{aligned}
& T_{e}=\int_{V_{e}} \frac{1}{2} \rho\left(\frac{\partial w}{\partial t}\right)^{2} \mathrm{~d} V, \quad U_{e}=\int_{V_{e}} \frac{1}{2} \sigma^{\mathrm{T}} \boldsymbol{\varepsilon} \mathrm{d} V, \\
& \delta W_{e}=\int_{A_{e}} \Delta p \delta w \mathrm{~d} A,
\end{aligned}
$$

where $\rho$ is the mass density of the panel, $V_{e}$ and $A_{e}$ are the volume and surface area of the element, and $\Delta p$ is the aerodynamic pressure computed by the supersonic piston theory and it can be evaluated by

$$
\Delta p=-\frac{2 q_{\infty}}{\beta}\left(\frac{M_{\infty}^{2}-2}{M_{\infty}^{2}-1} \frac{1}{U_{\infty}} \frac{\partial w}{\partial t}+\frac{\partial w}{\partial x}\right),
$$




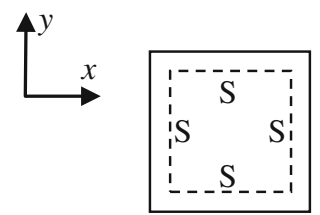

(a)

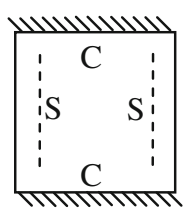

(b)

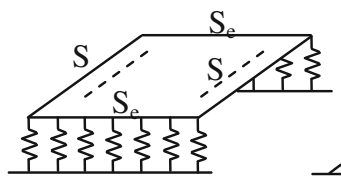

(d)

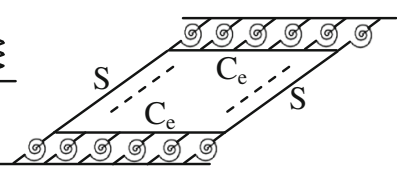

(e)
Fig. 2 Schematic diagrams of panels with different boundary conditions a SSSS b SCSC c SFSF d $\mathrm{SS}_{\mathrm{e}} \mathrm{SS}_{\mathrm{e}}$ e $\mathrm{SC}_{\mathrm{e}} \mathrm{SC}_{\mathrm{e}}$

where

$q_{\infty}=\frac{1}{2} \rho_{\infty} U_{\infty}^{2}, \quad \beta=\sqrt{M_{\infty}^{2}-1}$,

where $\rho_{\infty}, U_{\infty}$ and $M_{\infty}$ are the mass density, velocity and Mach number of the free airflow. In the analysis, a nondimensional aerodynamic pressure $\lambda=2 q_{\infty} a^{3} /(\beta D)$ is introduced in which $D=E h^{3} /\left[12\left(1-v^{2}\right)\right]$. In the case of $M_{\infty}>>1,\left[\left(M_{\infty}^{2}-2\right) /\left(M_{\infty}^{2}-1\right)\right]^{2} \mu / \beta \rightarrow \mu / M_{\infty}$, where $\mu=\rho_{\infty} a / \rho h$.

In this investigation, as it is shown in Fig. 2, SSSS, SCSC, and SFSF panels are researched, where " $\mathrm{S}$ ", "C" and "F" denote simply supported, clamped and free boundary conditions, respectively. Besides, the aeroelastic analysis of the $\mathrm{SS}_{\mathrm{e}} \mathrm{SS}_{\mathrm{e}}$ and $\mathrm{SC}_{\mathrm{e}} \mathrm{SC}_{\mathrm{e}}$ panels are also conducted, in which $\mathrm{S}_{\mathrm{e}}$ and $\mathrm{C}_{\mathrm{e}}$ represent the elastic simply supported and elastic clamped boundary conditions.

As far as the $\mathrm{SS}_{\mathrm{e}} \mathrm{SS}_{\mathrm{e}}$ panel is concerned, the virtual work of the element should be calculated by

$$
\begin{aligned}
& \delta W_{e}=\int_{A_{e}} \Delta p \delta w \mathrm{~d} A-\left.\left.\int_{l_{e}} k_{w} w\right|_{y=0} \delta w\right|_{y=0} \mathrm{~d} l, \text { or } \\
& \delta W_{e}=\int_{A_{e}} \Delta p \delta w \mathrm{~d} A-\left.\left.\int_{l_{e}} k_{w} w\right|_{y=b_{e}} \delta w\right|_{y=b_{e}} \mathrm{~d} l,
\end{aligned}
$$

where $k_{w}$ is the stiffness coefficient of the spring on the boundary of the panel, $b_{e}$ is the width of the element, and $l_{e}$ is the length of the element in the $x$ direction. As for the $\mathrm{SC}_{\mathrm{e}} \mathrm{SC}_{\mathrm{e}}$ panel, the virtual work can be obtained as

$$
\begin{aligned}
& \delta W_{e}=\int_{A_{e}} \Delta p \delta w \mathrm{~d} A-\left.\left.\int_{l_{e}} k_{\varphi} \frac{\partial w}{\partial y}\right|_{y=0} \delta \frac{\partial w}{\partial y}\right|_{y=0} \mathrm{~d} l, \text { or } \\
& \delta W_{e}=\int_{A_{e}} \Delta p \delta w \mathrm{~d} A-\left.\left.\int_{l_{e}} k_{\varphi} \frac{\partial w}{\partial y}\right|_{y=b_{e}} \delta \frac{\partial w}{\partial y}\right|_{y=b_{e}} \mathrm{~d} l,
\end{aligned}
$$

where $k_{\varphi}$ is the stiffness coefficient of the torsional spring on the boundary of the $\mathrm{SC}_{\mathrm{e}} \mathrm{SC}_{\mathrm{e}}$ panel.

In the AMM, the kinetic energy, strain energy and the virtual work of the panel are computed by the following expressions

$$
\begin{aligned}
& T=\frac{1}{2} \int_{V} \rho\left(\frac{\partial w}{\partial t}\right)^{2} d V, \\
& U=\frac{1}{2} \int_{V} \boldsymbol{\varepsilon}^{\mathrm{T}} \boldsymbol{\sigma} d V, \delta W=\int_{A} \Delta p \delta w \mathrm{~d} A,
\end{aligned}
$$

where $V$ and $A$ are the volume and surface area of the panel. Under the AMM, the transverse displacement $w$ is expressed as

$$
w(x, y, t)=\sum_{i=1}^{m} \sum_{j=1}^{n} \psi_{i j}(x, y) q_{i j}(t)=\psi^{\mathrm{T}}(x, y) \mathbf{q}(t),
$$

where $m$ and $n$ are the numbers of the assumed mode in the airflow direction ( $x$ direction) and $y$ direction, $\psi_{i j}$ is the assumed mode of the structural system, and according to different boundary conditions, the assumed modes are different. For the SSSS panel, the mode shape can be assumed as

$\psi_{i j}=\sin \frac{i \pi x}{a} \sin \frac{j \pi y}{b}$.

The assumed mode of the SCSC panel shown in Fig. 2b is given as

$$
\begin{aligned}
\psi_{i j}(x, y)=[ & \beta_{2 i j} \sin \beta_{1 i j} y-\beta_{1 i j} \sinh \beta_{2 i j} y \\
& \left.+\alpha_{i j}\left(\cos \beta_{1 i j} y-\cosh \beta_{2 i j} y\right)\right] \sin \frac{i \pi}{a} x,
\end{aligned}
$$

where

$$
\begin{aligned}
\beta_{1 i j} & =\sqrt{\chi_{i j}^{2}-\left(\frac{i \pi}{a}\right)^{2}}, \beta_{2 i j}=\sqrt{\chi_{i j}^{2}+\left(\frac{i \pi}{a}\right)^{2}}, \\
\alpha_{i j} & =\frac{\beta_{1 i j} \sinh \beta_{2 i j} b-\beta_{2 i j} \sin \beta_{1 i j} b}{\cos \beta_{1 i j} b-\cosh \beta_{2 i j} b},
\end{aligned}
$$

where $\chi_{i j}=\dot{\alpha}_{i j} / a$, and $\alpha_{i j}=a\left(\omega_{i j}\right)^{1 / 2}(\rho h / D)^{1 / 4}$ is the frequency parameter, in which $\omega_{i j}$ is the natural frequency of the panel. The assumed mode of the SFSF panel in Fig. 2c is given as

$$
\begin{aligned}
\psi_{i j}= & {\left[\beta_{2 i j} \xi_{i j} \sin \beta_{1 i j} y+\beta_{1 i j} \sinh \beta_{1 i j} y\right.} \\
& \left.-\alpha_{i j}\left(\eta_{i j} \cos \beta_{1 i j} y+\xi_{i j} \cosh \beta_{1 i j} y\right)\right] \sin \frac{i \pi x}{a},
\end{aligned}
$$

where

$$
\begin{aligned}
\xi_{i j} & =\frac{\chi_{i j}^{2} a^{2}}{i^{2} \pi^{2}}+v-1, \eta_{i j}=\frac{\chi_{i j}^{2} a^{2}}{i^{2} \pi^{2}}-v+1, \\
a_{i j} & =\frac{\xi_{i j} \beta_{2 i j} \sin \beta_{1 i j} b-\eta_{i j} \beta_{1 i j} \sin \beta_{2 i j} b}{\xi_{i j} \eta_{i j}\left(\cos \beta_{1 i j} b-\cosh \beta_{2 i j} b\right)} .
\end{aligned}
$$




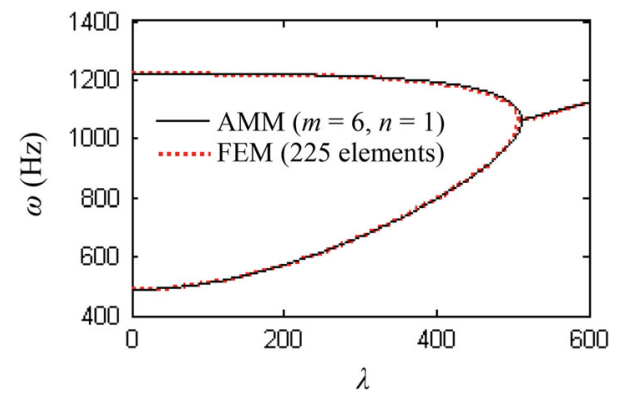

Fig. 3 Variations of natural frequency of the SSSS panel with aerodynamic pressure using different methods

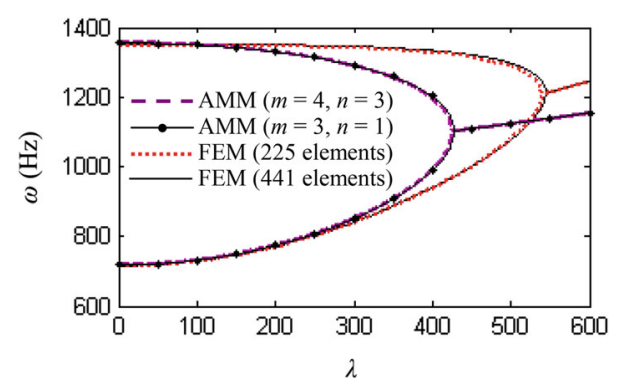

Fig. 4 Variations of natural frequency of the SCSC panel with aerodynamic pressure using different methods

As for the $\mathrm{SS}_{\mathrm{e}} \mathrm{SS}_{\mathrm{e}}$ panel, its mode shape can be assumed as

$$
\begin{aligned}
\psi_{i j}(x, y)= & \left(A_{1} \sin \beta_{1 i j} y+B_{1} \cos \beta_{1 i j} y+C_{1} \sinh \beta_{2 i j} y\right. \\
& \left.+D_{1} \cosh \beta_{2 i j} y\right) \sin \frac{i \pi}{a} x
\end{aligned}
$$

where $A_{1}, B_{1}, C_{1}$, and $D_{1}$ are the undetermined coefficients which can be determined by the boundary condition of the panel, and the boundary condition of the $\mathrm{SS}_{\mathrm{e}} \mathrm{SS}_{\mathrm{e}}$ panel can be expressed as follows

$y=0: M_{y}=-D\left(\frac{\partial^{2} w}{\partial y^{2}}+v \frac{\partial^{2} w}{\partial x^{2}}\right)=0$,

$V_{y}=-D\left[\frac{\partial^{3} w}{\partial y^{3}}+(2-v) \frac{\partial^{2} w}{\partial x^{2}}\right]=-k_{w} w$,

$y=b: M_{y}=-D\left(\frac{\partial^{2} w}{\partial y^{2}}+v \frac{\partial^{2} w}{\partial x^{2}}\right)=0$,

$V_{y}=-D\left[\frac{\partial^{3} w}{\partial y^{3}}+(2-v) \frac{\partial^{2} w}{\partial x^{2}}\right]=k_{w} w$,

where $M_{y}$ and $V_{y}$ are the bending moment and KelvinKirchhoff shear force resultant on the boundary. Substituting Eq. (19) into Eqs. (20a) and (20b), the homogenous equations about the coefficients $A_{1}, B_{1}, C_{1}$, and $D_{1}$ are obtained. By letting the coefficient determinant of the equations be equal to zero, the frequency equation of the panel is obtained. In addition, the coefficients $A_{1}, B_{1}, C_{1}$, and $D_{1}$ can be calculated as

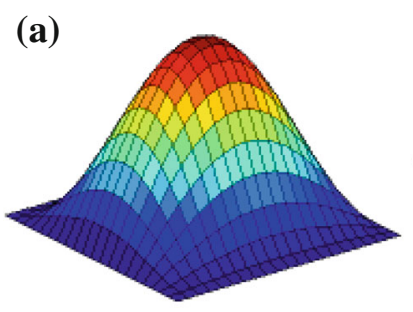

(b)
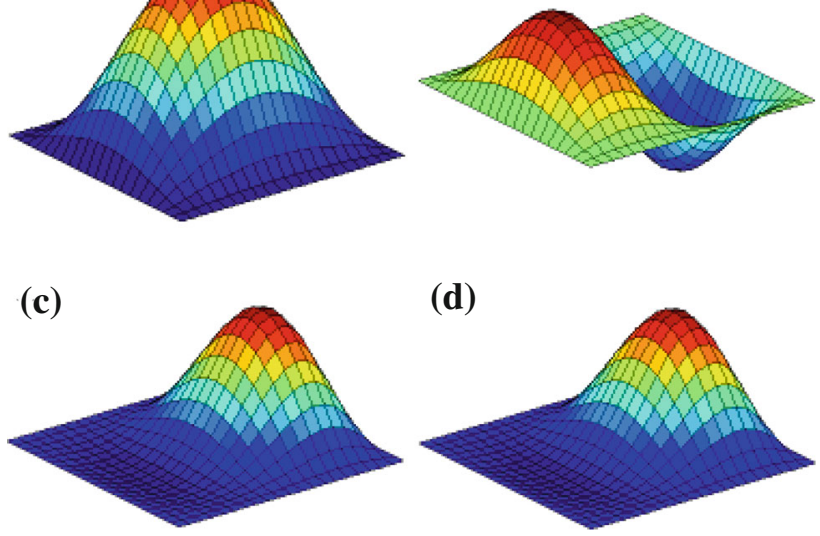

(d)

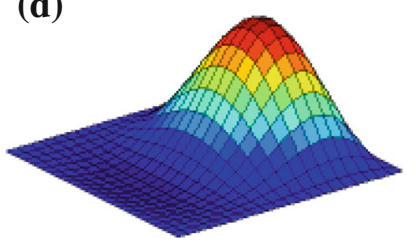

Fig. 5 First two orders of mode shape of the SCSC panel with and without aerodynamic pressure. $\mathbf{a} \lambda=0$, Mode $1 \mathbf{b} \lambda=0$, Mode 2 c $\lambda=546$, Mode $1 \mathbf{d} \lambda=546$, Mode 2

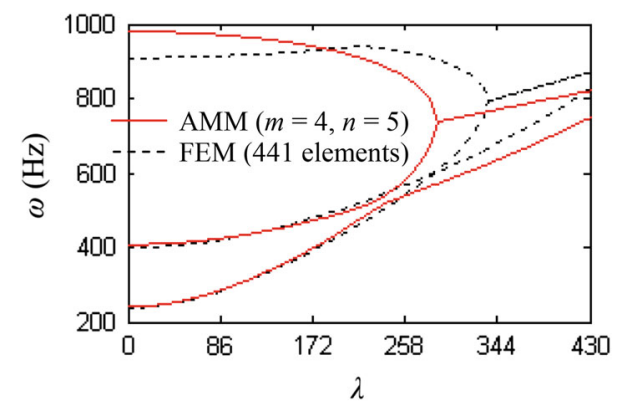

Fig. 6 Variations of natural frequency of the SFSF panel with aerodynamic pressure using different methods

$A_{1}=\frac{\frac{K_{w}}{D}\left(r_{1}+r_{2}\right) r_{2} \sinh \left(\beta_{2 i j} b\right)+g_{2} r_{1} r_{2}\left[\cos \left(\beta_{1 i j} b\right)-\cosh \left(\beta_{2 i j} b\right)\right]}{r_{1}\left[g_{1} r_{2} \sinh \left(\beta_{2 i j} b\right)-g_{2} r_{1} \sin \left(\beta_{1 i j} b\right)\right]}$,

$C_{1}=\frac{\frac{K_{w}}{D}\left(r_{1}+r_{2}\right) \sin \left(\beta_{1 i j} b\right)+g_{1} r_{2}\left[\cos \left(\beta_{1 i j} b\right)-\cosh \left(\beta_{2 i j} b\right)\right]}{g_{1} r_{2} \sinh \left(\beta_{2 i j} b\right)-g_{2} r_{1} \sin \left(\beta_{1 i j} b\right)}$,

$B_{1}=\frac{r_{2}}{r_{1}}, D_{1}=1$,

where

$g_{1}=\beta_{1 i j}\left[\beta_{1 i j}^{2}+(2-v) \frac{i^{2} \pi^{2}}{a^{2}}\right]$,

$g_{2}=\beta_{2 i j}\left[\beta_{2 i j}^{2}-(2-v) \frac{i^{2} \pi^{2}}{a^{2}}\right]$,

$r_{1}=\beta_{1 i j}^{2}+v \frac{i^{2} \pi^{2}}{a^{2}}, r_{2}=\beta_{2 i j}^{2}-v \frac{i^{2} \pi^{2}}{a^{2}}$,

$K_{w}=k_{w} b / D$.

Substituting Eqs. (1)-(3), (6) and (8) into Eqs. (7), (10) and (11), and the results into Eq. (4), after performing the calculation of variational, the element equation of motion can 
Fig. 7 First three orders of mode shape of the SFSF panel with and without aerodynamic pressure. $\mathbf{a} \lambda=0$, Mode $1 \mathbf{b}$ $\lambda=0$, Mode $2 \mathbf{c} \lambda=0$, Mode 3 $\mathbf{d} \lambda=336$, Mode 1 e $\lambda=336$, Mode $2 \mathbf{f} \lambda=336$, Mode 3

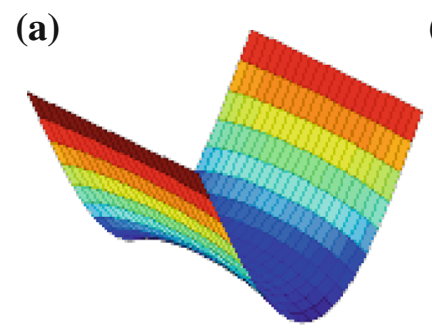

(b)

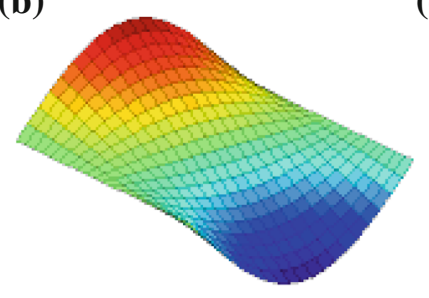

(c)

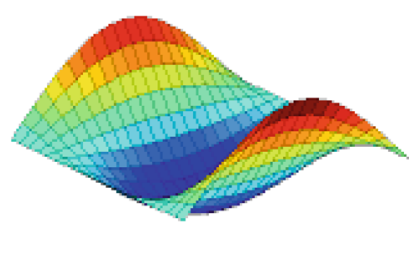

(d)

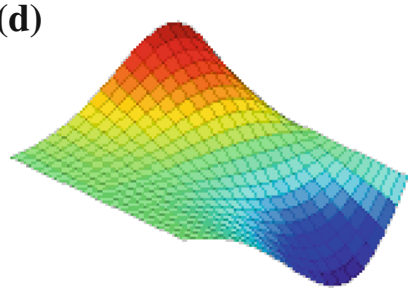

(e)

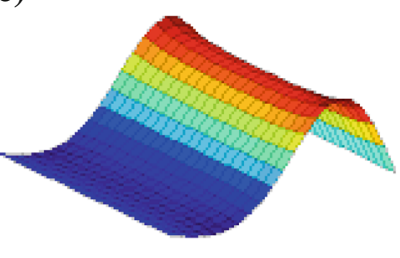

(f)

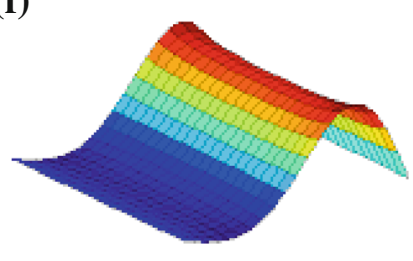

Table 1 Comparison of present natural frequency (Hz) with that computed by the ANSYS

\begin{tabular}{llllll}
\hline Various methods & \multicolumn{5}{l}{ Modes } \\
\cline { 2 - 6 } & 1 & 2 & 3 & 4 & 5 \\
\hline ANSYS & 240.31 & 399.92 & 912.33 & 975.82 & 1170.2 \\
Present results & 240.6 & 399.3 & 912.1 & 973.3 & 1162.2 \\
\hline
\end{tabular}

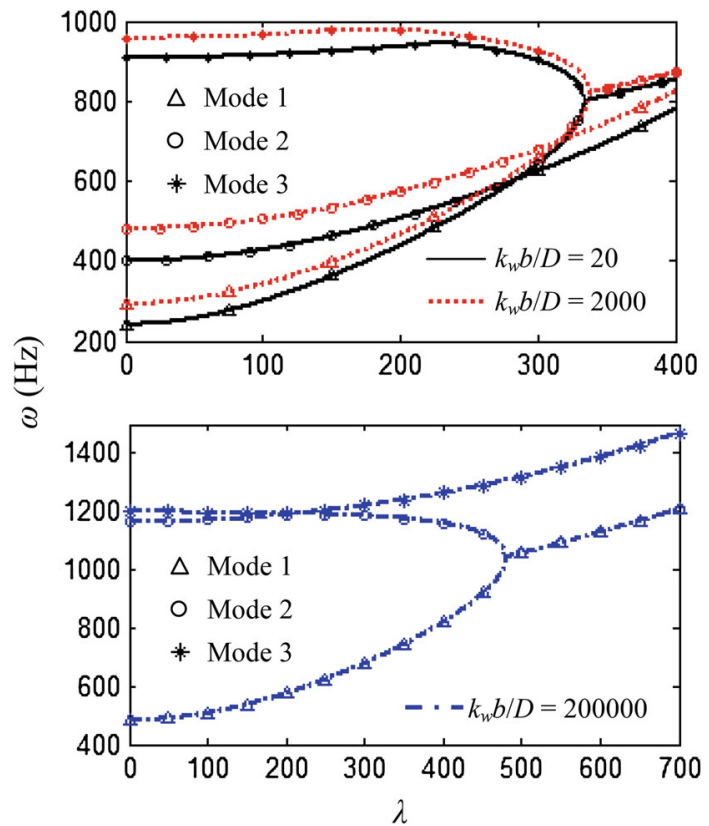

Fig. 8 Variations of natural frequencies of $\mathrm{SS}_{\mathrm{e}} \mathrm{SS}_{\mathrm{e}}$ panels with different boundary spring stiffness with the aerodynamic pressure

be obtained. By assembling the element matrices into global ones, the equation of motion of the whole panel is obtained. In the same way, substitution Eqs. (1)-(3), (8) and (13) into

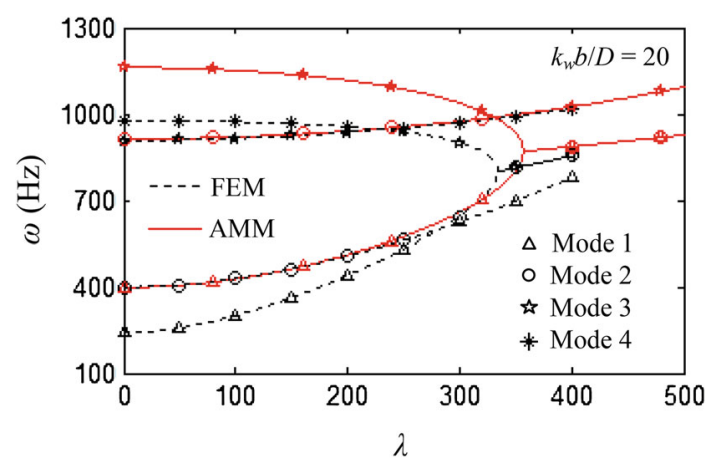

Fig. 9 Variations of natural frequencies of the $\mathrm{SS}_{\mathrm{e}} \mathrm{SS}_{\mathrm{e}}$ panel with the aerodynamic pressure obtained by different methods

Eq. (12) by means of Eqs. (14)-(22), and the results into Eq. (4), yields the equation of motion of the panel. Both the equations of motion obtained by the FEM and AMM can be expressed as follows

$\boldsymbol{M} \ddot{\boldsymbol{X}}+\boldsymbol{C}_{\Delta p} \dot{\boldsymbol{X}}+\left(\boldsymbol{K}+\boldsymbol{K}_{\Delta p}\right) \boldsymbol{X}=0$,

where $\boldsymbol{X}=\left[w_{1}, w_{1, y}, w_{1, x}, \ldots, w_{k}, w_{k, y}, w_{k, x}\right]^{\mathrm{T}}(k$ is the number of the node) is the nodal displacement vector in the FEM or $\boldsymbol{X}=\boldsymbol{q}^{\mathrm{T}}$ in the AMM, $\boldsymbol{M}$ is the mass matrix, $\boldsymbol{K}$ is the stiffness matrix, $\boldsymbol{C}_{\Delta p}$ is the aerodynamic damping matrix, and $\boldsymbol{K}_{\Delta p}$ is the aerodynamic stiffness matrix.

The general solution of Eq. (23) can be expressed as $\boldsymbol{X}=\boldsymbol{X}_{0} \exp (\Omega t)$, where $\boldsymbol{X}_{0}$ and $\Omega$ are the eigenvector and eigenvalue of the equation. Substitution of the general solution into Eq. (23) leads to the following eigenvalue problem of the structural system:

$$
\left[M \Omega^{2}+\boldsymbol{C}_{\Delta p} \Omega+\left(\boldsymbol{K}+\boldsymbol{K}_{\Delta p}\right)\right] \boldsymbol{X}_{0}=\mathbf{0} .
$$


Fig. 10 First three orders of mode shape of the $\mathrm{SS}_{\mathrm{e}} \mathrm{SS}_{\mathrm{e}}$ panel for $k_{w} b / D=20$ with and without aerodynamic pressure. a $\lambda=0$, Mode $1 \mathbf{b} \lambda=0$, Mode 2 c $\lambda=0$, Mode $3 \mathbf{d} \lambda=335$, Mode 1 e $\lambda=335$, Mode $2 \mathbf{f}$ $\lambda=335$, Mode 3 (a)

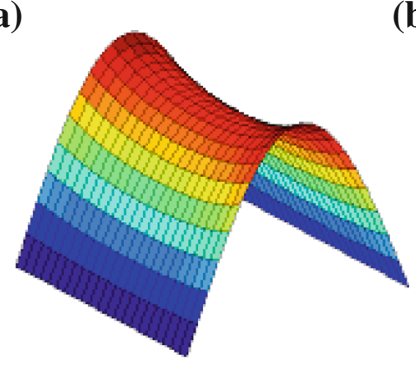

(d)

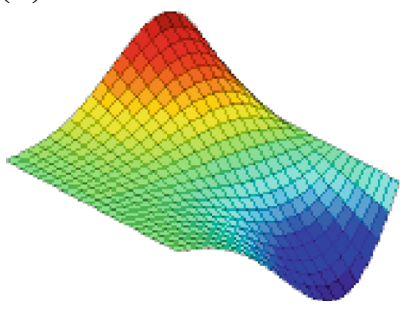

(b)

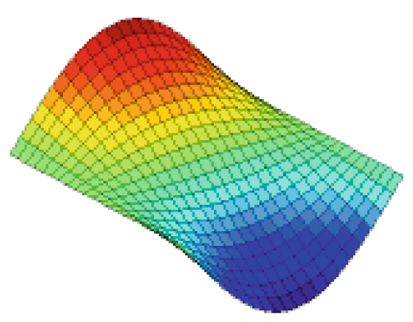

(e)

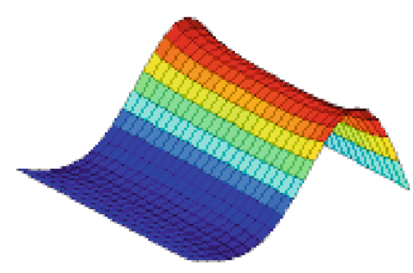

(c)

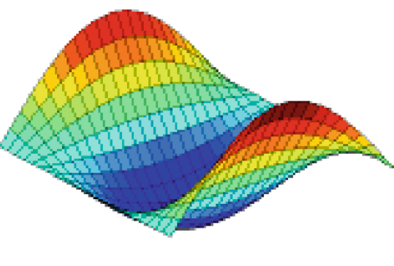

(f)

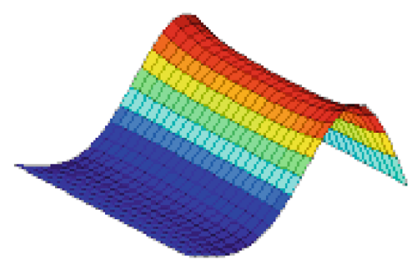

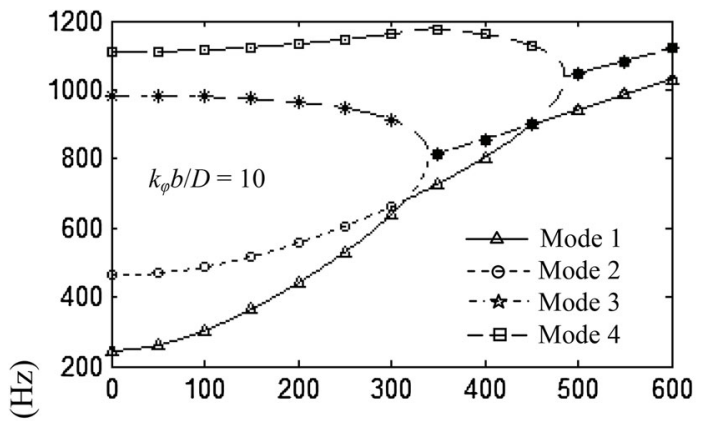

3

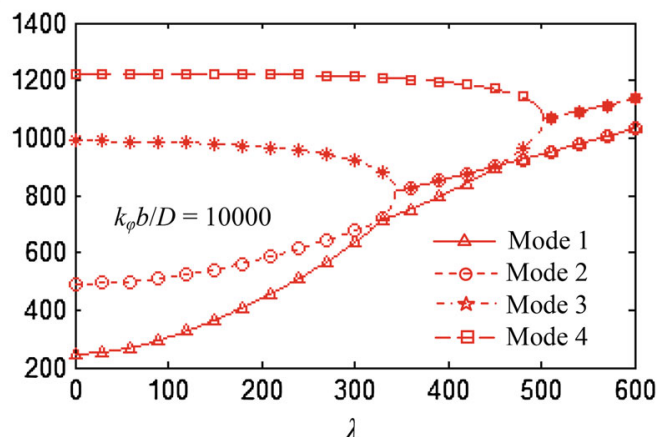

Fig. 11 Variations of natural frequencies of $\mathrm{SC}_{\mathrm{e}} \mathrm{SC}_{\mathrm{e}}$ panels with different boundary torsional spring stiffness with the aerodynamic pressure

The condition for getting a non-trivial solution of Eq. (24) requires the coefficient determinant to be zero

$$
\left|\boldsymbol{M} \boldsymbol{\Omega}^{2}+\boldsymbol{C}_{\Delta p} \Omega+\left(\boldsymbol{K}+\boldsymbol{K}_{\Delta p}\right)\right|=\mathbf{0} .
$$

Solving the eigenvalue problem, the complex eigenvalue can be obtained as $\Omega=i \omega+\Omega_{R}$, where $\omega$ is the natural frequency of the panel, and $\Omega_{R}$ is the real part of the eigenvalue. It is well known that the flutter will occur when the real part of the eigenvalue changes from negative to positive and usu- ally it can be estimated by the coalescence of two consecutive natural frequencies. The corresponding aerodynamic pressure $\lambda$ is called the critical flutter aerodynamic pressure which is denoted by $\lambda_{c r}$.

\section{Numerical simulations and discussions}

In this section, aeroelastic analysis of supersonic panels with different boundary conditions is carried out. The geometrical sizes and material properties of the panel are: $a=b=$ $0.1 \mathrm{~m}, h=0.001 \mathrm{~m} ; E=210 \mathrm{GPa}, v=0.33$ and $\rho=$ $7930 \mathrm{~kg} / \mathrm{m}^{3}$. The parameter $\mu / M_{\infty}$ used in the supersonic piston theory is equal to 0.01 .

First of all, the SSSS panel is studied. The variations of natural frequency with the aerodynamic pressure calculated by the AMM and FEM are shown in Fig. 3. It can be seen from the figure that the critical flutter aerodynamic pressure obtained by the two methods are nearly the same and they are both close to $\lambda=512$. It is also noted from Ref. [4] that the flutter bound of the fully simply supported isotropic panel is $\lambda_{c r}=512$. So it can be concluded that for the SSSS panel, AMM is accurate and efficient in the investigation on the flutter characteristics of the supersonic panel.

The flutter behaviors of the SCSC panel are then analyzed. Figure 4 shows the flutter bounds computed using different methods. It is seen from the figure that the convergences of the two methods are verified, and the critical flutter aerodynamic pressures obtained by the two methods are quite different, which are $\lambda_{c r}=428$ for AMM and 546 for FEM. However, the natural frequencies of the panel without aerodynamic pressure calculated by the two methods are equal, 
Fig. 12 First three orders of mode shape of the $\mathrm{SC}_{\mathrm{e}} \mathrm{SC}_{\mathrm{e}}$ panel with and without aerodynamic pressure. $\mathbf{a} \lambda=0$, Mode $1 \mathbf{b}$ $\lambda=0$, Mode $2 \mathbf{c} \lambda=0$, Mode 3 d $\lambda=400$, Mode 1 e $\lambda=400$, Mode $2 \mathbf{f} \lambda=400$, Mode 3

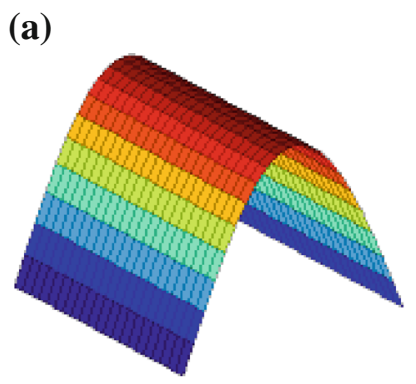

(b)

(c)
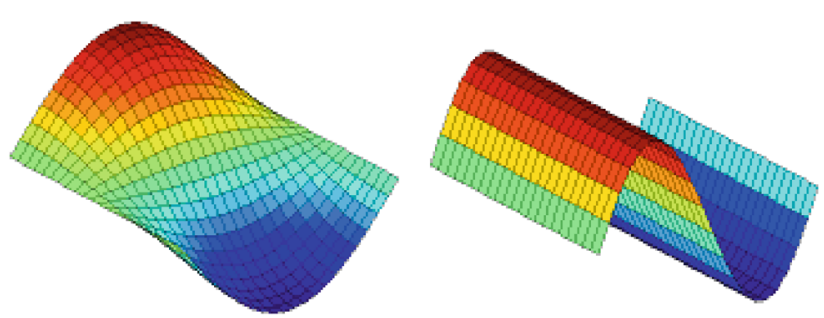

(d)

(e)

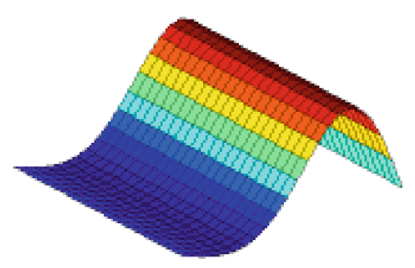

(f)
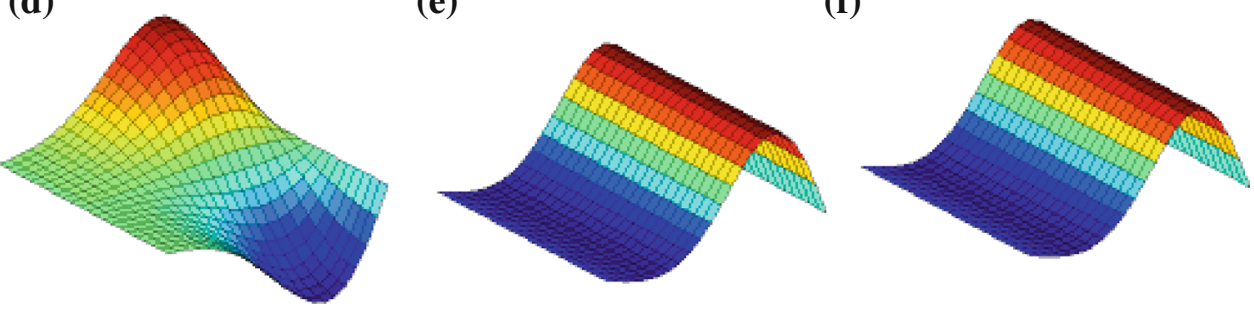

and with the increase of the aerodynamic pressure, the differences of the results obtained by these two methods increase. It is indicated that the AMM will produce error in the aeroelastic analysis of the panels with the SCSC boundary condition. The mode shapes of the SCSC panel with and without aerodynamic pressure are calculated using the FEM and shown in Fig. 5. It is obviously observed from the figure that under the aerodynamic pressure, the mode shapes of the panel are changed, and at the flutter bound, the first two orders of mode shape are the same.

Panels with two free ends are also studied in this investigation. Aeroelastic analysis result is shown in Fig. 6. It is seen from the figure that in the AMM, to get a similar result to that of the FEM, 20 orders of the assumed mode (four orders in the $x$ direction and five orders in the $y$ direction) are used. Nevertheless, the flutter bounds obtained by the two methods are still quite different. According to the result of the FEM, the flutter occurs at the coupling of the second and third orders of natural frequency, and the critical flutter aerodynamic pressure is $\lambda_{c r}=336$. Figure 7 displays the mode shapes of the panel with and without aerodynamic pressure. It is observed that at the flutter bound, the first flutter mode is similar to the second order of the mode without aerodynamic pressure, but the position of the mode's peak is changed from $x=0.5 a$ to $x=0.75 a$.

Next, the aeroelastic characteristics of the panels with nonclassical boundary conditions are investigated. First of all, the $\mathrm{SS}_{\mathrm{e}} \mathrm{SS}_{\mathrm{e}}$ panel is studied. Prior to the main topic of aeroelastic analysis, the correctness of the methodology for formulating the equation of motion of the panel with this type of boundary condition is verified by comparing the present natural frequency with that obtained by the ANSYS. The results are shown in Table 1. It is seen from the table that the natural frequency obtained in this paper agrees well with that cal- culated by the ANSYS, which indicates that the equation of motion formulated in this study is correct.

Based on the verification, aeroelastic analysis of the $\mathrm{SS}_{\mathrm{e}} \mathrm{SS}_{\mathrm{e}}$ panel is carried out. Figure 8 displays the variations of natural frequencies of the $\mathrm{SS}_{\mathrm{e}} \mathrm{SS}_{\mathrm{e}}$ panels with different boundary spring stiffness with the aerodynamic pressure using the FEM. It is seen from the figure that when the boundary of the panel is elastically restrained, the veering of the natural frequency occurs. For instance, the first order of natural frequency of the panel with $k_{w} b / D=20$ veers near the non-dimensional aerodynamic pressure $\lambda=300$, and the third order of natural frequency of the panel with $k_{w} b / D=200000$ veers at $\lambda=200$. Moreover, it is observed from the figure that with the increase of the boundary spring stiffness, the critical flutter aerodynamic pressure increases and is close to that of the SCSC panels.

The AMM is also used in the aeroelastic analysis of the $\mathrm{SS}_{\mathrm{e}} \mathrm{SS}_{\mathrm{e}}$ panel for $k_{w} b / D=20$. Variation of natural frequency of the panel with the aerodynamic pressure calculated by the AMM is shown in Fig. 9. It is noted that the first order of natural frequency is lost in the AMM, and the critical flutter aerodynamic pressure is larger than that obtained through the FEM. Consequently, it can be concluded that the AMM is not as precise as the FEM in the aeroelastic analysis of the $\mathrm{SS}_{\mathrm{e}} \mathrm{SS}_{\mathrm{e}}$ panel.

The mode shapes of the $\mathrm{SS}_{\mathrm{e}} \mathrm{SS}_{\mathrm{e}}$ panel for $k_{w} b / D=20$ with and without aerodynamic pressure are displayed in Fig. 10. It can also be observed from the figure that at the flutter bound, the first fluttering mode is similar to the second order of the mode without aerodynamic pressure, and the position of the mode's peak is still changed.

Since the AMM will produce error in the aeroelastic analysis of panels with elastically restrained boundary condition, FEM is applied to investigate the flutter characteristics of 
the $\mathrm{SC}_{\mathrm{e}} \mathrm{SC}_{\mathrm{e}}$ panels. Figure 11 shows the variations of natural frequency of the $\mathrm{SC}_{\mathrm{e}} \mathrm{SC}_{\mathrm{e}}$ panels with different boundary torsional spring stiffness with the aerodynamic pressure. It can be seen from the figure that the mode veering of the $\mathrm{SC}_{\mathrm{e}} \mathrm{SC}_{\mathrm{e}}$ panel is more obvious than that of the $\mathrm{SS}_{\mathrm{e}} \mathrm{SS}_{\mathrm{e}}$ panel. In addition, it can also be noted that with the increase of the spring stiffness, the critical flutter aerodynamic pressure increases, but the increase is very slow. The mode shape of the $\mathrm{SC}_{\mathrm{e}} \mathrm{SC}_{\mathrm{e}}$ panel is also computed by the FEM as shown in Fig. 12. The same conclusion can be drawn as previous.

\section{Conclusions}

The flutter properties of supersonic panels with different boundary conditions are analyzed. The classical thin plate theory is applied, and the piston theory is used to evaluate the unsteady aerodynamic pressure. The equation of motion is formulated using the Hamilton's principle, and the AAM and FEM are used to obtain the discrete equation of motion. Aeroelastic characteristics of the panel with different boundary conditions are investigated. The special flutter properties of panels with elastically restrained boundary condition are also analysed. The accuracy of the AMM in the flutter analysis of panels with different constraints is researched. From the numerical results, the following conclusions can be drawn:

(1) AMM is not as precise as the FEM in the aeroelastic analysis of supersonic panels.

(2) Mode veering will occur when the boundary of the structure is elastically restrained.

(3) The first mode of the fluttering panel is usually similar to the second mode shape of the panel without aerodynamic pressure, and the position of the flutter mode's peak is usually changed from $x=0.5 a$ to $x=0.75 a$.

(4) The stronger the boundary constraint, the stronger the aeroelastic stability of the supersonic panel.

Acknowledgments This research is supported by the National Basic Research Program of China (No. 2011CB711100) and the National Natural Science Foundation of China (Nos. 10672017, 11172084).

\section{References}

1. Dowell EH (1966) Nonlinear oscillations of a fluttering plate I. AIAA J 4(7):1267-1275

2. Dowell EH (1967) Nonlinear oscillations of a fluttering plate II. AIAA J 5(10):1856-1862
3. Dowell EH (1970) Panel flutter: a review of the aeroelastic stability of plates and shells. AIAA J 8:386-399

4. Guo X, Mei C (2006) Application of aeroelastic modes on nonlinear supersonic panel flutter at elevated temperatures. Comput Struct 84:1619-1628

5. Kouchakzadeh MA, Rasekh M, Haddadpour H (2010) Panel flutter analysis of general laminated composite plates. Compos Struct 92:2906-2915

6. Navazi HM, Haddadpour H (2011) Nonlinear aero-thermoelastic analysis of homogeneous and functionally graded plates in supersonic airflow using coupled models. Compos Struct 93:2554-2565

7. Song ZG, Li FM (2011) Active aeroelastic flutter analysis and vibration control of supersonic beams using the piezoelectric actuator/sensor pairs. Smart Mater Struct 20:055013

8. Li FM, Chen ZB, Cao DQ (2011) Improving the aeroelastic flutter characteristics of supersonic beams using piezoelectric material. $\mathrm{J}$ Intell Mater Syst Struct 22:615-629

9. Song ZG, Li FM (2012) Active aeroelastic flutter analysis and vibration control of supersonic composite laminated plate. Compos Struct 94:702-713

10. Song ZG, Li FM (2013) Optimal locations of piezoelectric actuators and sensors for supersonic flutter control of composite laminated panels. J Vib Control. doi:10.1177/1077546313480538

11. Gray CE, Mei C, Shore CP (1990) Finite element method for largeamplitude two-dimensional panel flutter at hypersonic speeds. AIAA J 29(2):290-298

12. Zhou RC, Xue DY, Mei C (1994) Finite element time domainmodal formulation for nonlinear flutter of composite panels. AIAA J 32:2044-2052

13. Han AD, Yang TY (1983) Nonlinear panel flutter using high-order triangular finite element. AIAA J 21:1453-1461

14. Li QQ, Mei C, Huang JK (2007) Suppression of thermal postbckling and nonlinear panel flutter motions using piezoelectric actuators. AIAA J 45(8):1861-1873

15. Koo KN, Hwang WS (2004) Effects of hysteretic and aerodynamic damping on supersonic panel flutter of composite plates. J Sound Vib 273:569-583

16. Moon SH (2006) Finite element analysis and design of control system with feedback output using piezoelectric sensor/actuator for panel flutter suppression. Finite Elem Anal Des 42:1071-1078

17. Moon SH, Kim SJ (2003) Suppression of nonlinear composite panel flutter with active/passive hybrid piezoelectric networks using finite element method. Compos Struct 59:525-533

18. Xue DY, Mei C (1993) Finite element nonlinear flutter and fatigue life of two-dimensional panels with temperature effects. J Aircr 3(6):993-1000

19. Li FM, Song ZG (2013) Flutter and thermal buckling control for composite laminated panels in supersonic flow. J Sound Vib 332(22):5678-5695 